\title{
Le racisme institutionnel dans les attributions de logement social. Une comparaison franco-britannique.
}

Article paru dans Hommes et migrations, n 1264, novembre-décembre 2006, pp. 103-112.

\section{Résumé :}

Cet article vise à questionner l'existence d'un modèle français républicain d'intégration, théoriquement aveugle aux différences ethniques, en étudiant la gestion locale des attributions de logements sociaux et à travers un détour comparatif par la Grande-Bretagne. La comparaison des politiques et pratiques locales d'attribution à Marseille et Birmingham conduit à mettre en évidence l'impact des idéologies nationales sur la mise en forme politique locale des enjeux mais aussi les contradictions de ces mises en forme et l'existence d'un racisme institutionnel des deux côtés de la Manche. 


\section{Le racisme institutionnel dans les attributions de logement social.}

\section{Une comparaison franco-britannique}

La production de frontières ethniques jusqu'au cœur des institutions républicaines (éducation, logement, police, etc.), dans une société qui s'aveugle volontairement sur sa propre différenciation ethnique, est la grande contradiction du «modèle français de discrimination ${ }^{1}$. La politique du logement social illustre parfaitement cette contradiction. Formulée au plan national dans des catégories universalistes («droit au logement» et «mixité sociale»), sans jamais aborder de front la question des «immigrés» ou des «minorités ethniques $»^{2}$, elle est marquée au plan local par la prégnance des catégorisations ethniques dans la gestion quotidienne du logement social ${ }^{3}$. Comment expliquer cette saillance de l'ethnicité dans les représentations et les pratiques d'attribution des acteurs HLM ? Dans quelle mesure remet-elle en question la référence à un modèle français républicain d'intégration?

Ce sont ces questions qui nous ont conduit à éclairer le cas français par le cas britannique, par une traversée du miroir qui nous mènera à Birmingham pour revenir ensuite à Marseille. On sait les contextes nationaux contrastés, modèle républicain d'un côté, communautariste ou multiculturaliste de l'autre ${ }^{4}$. La reconnaissance de longue date d'un racisme institutionnel outre-Manche tranche avec des représentations sociales arc-boutées en France sur la dimension individuelle et intentionnelle des discriminations, lorsque celles-ci n'y sont pas purement et simplement niées. Le détour comparatif que nous proposons vise à apporter un double éclairage : sur les effets des politiques anti-discrimination en

\footnotetext{
${ }^{1}$ Mouvements, «Le modèle français de discrimination. Un nouveau défi pour l'antiracisme », $\mathrm{n}^{\circ} 4$, mai-juillet 1999 ; H\&M, «Connaittre et combattre les discriminations », $\mathrm{n}^{\circ}$ 1219, mai-juin 1999 ; V. De Rudder, C. Poiret, F. Vourc'h, L'Inégalité raciste. L'universalité républicaine à l'épreuve, Paris, PUF, 2000.

${ }^{2}$ Nous désignons par le terme d'immigré, non pas la catégorie juridique, mais la figure sociale de l'immigré, ce qui inclut notamment tous les descendants d'immigrés qui ont la nationalité française et sont stigmatisés en raison de leur «origine ». Nous définissons les minorités ethniques dans une perspective constructiviste comme des groupes minorisés, c'est-à-dire subissant un rapport de domination au sein de la société du fait de leur origine réelle ou supposée.

${ }^{3}$ GELD, Les Discriminations raciales et ethniques dans l'accès au logement social, Paris, GELD, 2001 ; T. Kirszbaum, "Les immigrés dans les politiques de l'habitat. Variations locales sur le thème de la diversité », Sociétés contemporaines, $\mathrm{n}^{\circ}$ 33-34, 1999, pp. 87-110.

${ }^{4}$ E. Bleich, Race politics in Britain and France: ideas and policy-making since the 1960s, Cambridge, Cambridge University Press, 2003; D. Lapeyronnie, L'Individu et les minorités. La France et la GrandeBretagne face à leurs immigrés, Paris, PUF, 1993; C. Neveu, Communauté, nationalité et citoyenneté. De l'autre côté du miroir, les Bangladeshis de Londres, Paris, Karthala, 1993.
} 
Grande-Bretagne tout d'abord, à travers la comparaison du racisme institutionnel dans les attributions de logement social à Birmingham hier (avant les années 1980) et aujourd'hui ; sur l'opposition des modèles nationaux ensuite, à travers la comparaison de ces phénomènes à Marseille et Birmingham aujourd'hui. Ce détour géographique se veut aussi théorique. Alors que la «pensée d'Etat» républicaine sur l'intégration et l'immigration ${ }^{5}$ a longtemps fait obstacle à une sociologie française des phénomènes discriminatoires, on entrevoit aujourd'hui tout le bénéfice à tirer des théories anglosaxonnes et notamment du concept de racisme institutionnel ${ }^{6}$. Ce concept proposé dès les années 1960 aux Etats-Unis par les théoriciens du Black Power ${ }^{7}$ et très tôt importé en Grande-Bretagne suggère d'envisager les discriminations ethniques comme le produit de logiques, de contraintes, de routines, d'une culture, institutionnels. Le GELD se situe clairement dans cette perspective lorsqu'il affirme que, "Loin de se réduire aux seules discriminations intentionnelles, (...) les traitements inégalitaires sont principalement le fruit d'un système local auquel participe une multitude d'institutions installées dans des routines gestionnaires et pas toujours conscientes des effets produits par la culture de la norme implicite $»^{8}$. Loin d'être une aberration raciste, la saillance de l'ethnicité dans les représentations des acteurs prend alors tout sens rapportée à leur univers institutionnel.

Méthodologiquement, cette recherche s'appuie sur des entretiens conduits auprès des divers acteurs en charge des attributions de logement social à Birmingham et Marseille. Ces deux métropoles à tradition industrielle ont joué, en particulier après la seconde guerre mondiale, un rôle de premier plan dans l'accueil des immigrés (Indiens, Pakistanais, Bangladeshis, Afro-antillais, mais aussi Irlandais à Birmingham ; immigrés originaires du Maghreb et d'Afrique noire à Marseille, où se sont en outre établis un nombre substantiel de Comoriens depuis les années 1980). Ces communautés s'y inscrivent fortement dans l'espace urbain (inner city à Birmingham, «quartiers nord » de logement social et centre ancien de Marseille). Leurs parcours résidentiels sont intimement liés aux mécanismes locaux d'accès au - et d'exclusion du - logement social. Les deux villes ont traversé depuis les années 1970 une crise économique et urbaine, à laquelle elles ont réagi par de nouvelles stratégies d'attractivité. Ces politiques, le contexte socio-économique plus général ainsi que la libéralisation du secteur du logement social dans les deux pays ont accentué la pression sur le marché du logement.

\footnotetext{
${ }^{5}$ A. Sayad, La Double Absence : des illusions de l'émigré aux souffrances de l'immigré, Paris, Seuil, 1999.

${ }^{6} \mathrm{M}$. Wieviorka, «La production institutionnelle du racisme », H\&M, $\mathrm{n}^{\circ} 1211$, janvier-février, 1998, pp. 5-15.

${ }^{7}$ S. Carmichael, C.V. Hamilton, Black power : the politics of liberation in America, New York, Vintage Books, 1967.

${ }^{8}$ Op. cit., p. 3.
} 


\section{La traversée du miroir : le racisme institutionnel dans les attributions de logement social à Birmingham}

Les discriminations ethniques dans l'accès au logement municipal, une histoire ancienne

Le débat britannique sur les inégalités ethniques des conditions de logement s'est noué dès les années 1960 autour d'un conflit d'interprétations désigné comme le «débat choix -contrainte »" , opposant les explications par les choix individuels des ménages, supposés culturellement différenciés, aux explications par les discriminations ethniques. Deux grandes recherches ayant pour cadre Birmingham ont défendu la thèse de la contrainte en montrant l'existence de mécanismes discriminatoires. Tout d'abord, Rex et son équipe ${ }^{10}$ montrent qu'en imposant une règle d'éligibilité apparemment neutre d'un minimum de cinq ans de résidence à Birmingham, la municipalité ferme de fait l'accès de son parc à tous les nouveaux migrants, produisant en cela une discrimination indirecte. Ensuite, quelques années plus tard, dans une enquête réalisée à la fin des années 1970, Henderson et Karn tentent de comprendre la persistance des inégalités malgré la relaxation de la qualification résidentielle ${ }^{11}$. Dépassant l'analyse des politiques formelles, ils montrent que la stratégie municipale d'attribution est marquée par les contradictions entre l'objectif officiel de satisfaction du besoin en logement et les objectifs officieux de réduction de la vacance et de prévention des conflits de voisinage, en vue de minimiser les coûts de gestion. Ces contradictions conduisent à une recatégorisation informelle des candidats selon le critère de la «respectabilité » du ménage, les catégories de race et de classe étant mobilisées, consciemment ou non, comme des indices de cette respectabilité. Henderson et Karn en concluent que «les pratiques discriminatoires sont un produit de la structure normale des attributions, et que cette structure est telle qu'elle satisfait certains intérêts dans un schéma beaucoup plus large de compétition pour des ressources rares dans notre société » (p. 275). Si les quelques études récemment publiées sur ce thème tendent à montrer la persistance du racisme institutionnel malgré les politiques d'égalité mises en place ${ }^{12}$, leur rareté laisse ouvertes de nombreuses questions de recherche. Qu'ont changé concrètement ces politiques ?

\footnotetext{
${ }^{9}$ R. Tomlins, Housing experiences of minority ethnic communities in Britain : an academic literature review and annotated bibliography, CRER, University of Warwick, 1999. Aujourd'hui, la plupart des auteurs s'accordent à considérer ces inégalités comme un complexe mélange d'éléments de choix et de contraintes. Cf. P. Sarre, D. Phillips, R. Skellington, Ethnic minority housing : explanations and policies, Aldershot, Avebury, 1989.

${ }^{10}$ J. Rex, D. Moore, Race, community and conflict : a study of Sparkbrook, Londres, OUP, 1967 ; J. Rex, S. Tomlinson (avec D. Hearnden et P. Ratcliffe), Colonial immigrants in a British city. A class analysis, Londres, Routledge, 1979.

${ }^{11} \mathrm{~J}$. Henderson, V. Karn, Race, class and state housing : inequality and the allocation of public housing in Britain, Aldershot, Gower, 1987.

${ }^{12}$ S. Jeffers, P. Hoggett, «Like counting deckchairs on the Titanic : a study of institutional racism and housing allocations in Haringey and Lambeth », Housing studies, 10, 3, 1995, pp. 325-344 ; S. Halliday, "Institutional racism in bureaucratic decision-making: a case study in the administration of homelessness law", Journal of law and society, Vol. 27, $\mathrm{n}^{\circ} 3$, septembre 2000, pp. 449-471.
} 
Les années 1980 ont été le témoin d'un fort développement des politiques locales de lutte contre les discriminations, notamment dans les grandes municipalités de gauche comme Birmingham, et simultanément de réformes nationales radicales de privatisation du logement social, aux dépens des groupes qui dépendent le plus de ce secteur, notamment les minorités ethniques ${ }^{13}$. A Birmingham, le parc municipal est passé de 120000 à 80000 logements suite à la vente aux locataires et aux transferts partiels de stock à des associations de logement, les locataires ayant voté en 2002 contre un transfert global. Par ailleurs, une quarantaine d'associations de logement privées y gèrent aujourd'hui environ 40000 logements sociaux et ont développé des politiques d'égalité raciale très similaires à celle de la municipalité.

Depuis les années 1980, la municipalité a affiché un fort volontarisme en matière d'égalité raciale et notamment d'accès des minorités ethniques au logement social. Néanmoins, trois éléments dans la mise en forme politique locale du problème vont dans le sens d'une euphémisation du racisme institutionnel. Tout d'abord, les inégalités sont largement interprétées comme le résultat de «besoins différents » des minorités ethniques, lesquels ne sont définis nulle part. Ensuite, c'est le racisme individuel, celui des locataires white (désigné comme «harcèlement racial» ou «comportement antisocial») qui est présenté comme l'autre grande cause du problème. Enfin, la concentration ethnico-spatiale n'est pas considérée en elle-même comme problématique. Elle est considérée comme légitime à partir du moment où elle est choisie par les minorités ethniques, et illégitime à partir du moment où elle est subie. Toute politique coercitive de spatialisation des groupes ethniques sur le territoire urbain est ainsi perçue comme illégitime et illégale, en tant qu'elle contrevient tant à l'exercice du choix individuel qu'au principe de non discrimination. Cette construction du problème aboutit à un traitement finalement très partiel du racisme institutionnel. La politique municipale met plutôt l'accent sur deux objectifs, la prise en compte des «besoins spécifiques » des minorités ethniques (développement de recherches sur les aspirations des minorités ethniques et d'une politique de communication dans leur direction, etc.) et la lutte contre le « harcèlement racial ». Quant à la lutte contre les discriminations institutionnelles, elle se réduit pour l'essentiel à l'amélioration de la représentation des minorités ethniques au sein du personnel, ce qui reflète des présupposés multiculturalistes selon lesquels les groupes ethniques minoritaires sont toujours mieux défendus par eux-mêmes.

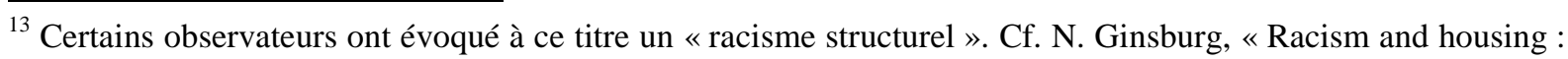
concepts and reality », in P. Braham, A. Rattansi et R. Skellington (dir.), Racism and anti-racism, Londres, Sage, 1992, pp. 109-132.
} 
A côté des politiques locales d'égalité raciale, l'un des éléments d'action publique les plus originaux a été, au plan national, le soutien donné dans les années 1980 par la Corporation du logement (quango régulant les associations de logement) à la création et au développement d'associations de logement black, définies comme ayant plus de $80 \%$ de minorités ethniques dans leur conseil d'administration. Cette politique repose sur ce même présupposé multiculturaliste et sur l'idéologie de l'empowerment communautaire ${ }^{14}$. L'expérience de Birmingham conduit toutefois à pointer les limites de ces associations, la plus évidente étant leur poids relatif (moins de 2,5\% du parc total de logements). Une deuxième limite est la remise en question de l'indépendance et de la viabilité de ces associations alors que la Corporation du logement leur a brusquement retiré son soutien dans les années 1990, délaissant l'impératif de diversité au profit de l'efficacité et de la concentration du secteur. Dans ce contexte, nombre des associations de logement black sont contraintes de joindre de plus grandes associations de logement (mainstream) pour survivre, aux dépens de leur indépendance et de leur défense d'une identité ethnique spécifique. Enfin, troisième grande limite, ces associations ont assis leur légitimité sur la référence aux «besoins spécifiques », avec le risque de renforcer des visions essentialisantes du problème. Pour toutes ces raisons, ces associations apparaissent difficilement comme une solution aux discriminations ethniques dans l'accès au logement social.

\section{Des effets en demi-teinte sur le racisme institutionnel}

La municipalité demeure ainsi aujourd'hui le principal bailleur social pour les minorités ethniques de Birmingham, ce qui conduit à soulever la question des résultats de sa politique antidiscrimination. Henderson et Karn concluaient de leur étude que la lutte contre les discriminations impliquait de "reconsidérer tout le fondement des attributions, et en particulier l'accent mis sur la réduction du taux de vacance » (p. 279). Or la politique anti-discrimination de la municipalité n'a pas remis en cause les fondements des attributions. Certains des changements introduits (monitoring ethnique, recrutement d'agents d'appartenance ethnique minoritaire, etc.) ont semble-t-il permis d'agir sur les représentations des acteurs et de réduire le racisme institutionnel. Ainsi, au cours de nos entretiens, nous avons entendu peu de stéréotypes ethniques ouvertement négatifs et la plupart des acteurs reconnaissaient l'existence de discriminations. Pourtant, le monitoring révèle la perpétuation du désavantage ethnique et l'analyse des politiques et pratiques d'attribution conduit à pointer la persistance et le renouvellement du racisme institutionnel. On constate tout d'abord la permanence des conflits d'objectifs entre satisfaction des besoins en logement, réduction de la vacance et évitement des conflits de voisinage. En outre, l'émergence au plan national d'un cadrage du problème des attributions en termes de choix, avec l'objectif de remplacer à l'horizon 2010 les systèmes de points par des «systèmes de location fondés sur le choix » (choice-based lettings systems) selon lesquels les

\footnotetext{
${ }^{14}$ M. Harrison, Housing, 'race', social policy and empowerment, Aldershot, Avebury, 1995 ; Corporation du logement, Five Year Programme for BME housing associations, Londres, Corporation du logement, 1986.
} 
usagers seraient invités à répondre aux annonces publiées par les bailleurs sociaux, fragilise la portée de la reconnaissance du besoin en logement comme fondement des attributions ${ }^{15}$.

En dépit de leurs effets positifs, les politiques anti-discriminatoires elles-mêmes introduisent paradoxalement de nouvelles sources de racisme institutionnel. Le traitement du harcèlement racial illustre parfaitement ces contradictions. La politique municipale préconise d'éviter de loger des ménages d'appartenance ethnique minoritaire dans les quartiers de logement social de l'outer city à dominante white considérés comme hostiles aux minorités ethniques (no go areas). Cette politique conduit paradoxalement à reproduire la concentration des minorités ethniques dans l'inner city et par conséquent dans les logements et quartiers les plus dévalorisés. D’une façon plus générale, l'un des principaux vecteurs de racisme indirect tient dans le stéréotype toujours en vigueur, y compris chez des agents d'appartenance ethnique minoritaire, selon lequel les minorités ethniques préfèreraient vivre dans l'inner city. Ce stéréotype est d'autant plus prégnant qu'il s'articule étroitement aux intérêts des acteurs du logement social et sert leur objectif de réduction de la vacance. Pourtant, la municipalité elle-même a produit en 1998 un rapport qui attestait d'un désir croissant des minorités ethniques, en particulier des jeunes ménages, de vivre hors de l'inner city ${ }^{16}$.

\section{Marseille au miroir de Birmingham : idéologie républicaine et racisme institutionnel}

En France, les systèmes locaux du logement social englobent une multitude d'acteurs au-delà des organismes HLM, dont beaucoup interviennent dans le processus d'attribution ${ }^{17}$. Nous nous focaliserons ici, comme à Birmingham, sur les bailleurs sociaux, qui maintiennent une forte autonomie d'action sur les attributions. Si les organismes HLM constituent un monde pluriel (sociétés anonymes, offices publics, SEM, etc.), notre enquête conduit à mettre en évidence de fortes similitudes dans la présence et les fondements du racisme institutionnel d'un organisme à l'autre.

\section{Idéologie républicaine et déni des discriminations}

La traversé du miroir nous renvoie d'abord l'image d'une différence fondamentale dans la construction du problème : si à Birmingham, l'existence d'un racisme institutionnel est reconnue, en dépit de toutes les nuances apportées ci-dessus, elle fait à Marseille l'objet d'un véritable déni. Seuls le FASILD et certaines associations semblent localement porteurs d'une représentation du problème

\footnotetext{
${ }^{15}$ Office of the Deputy Prime Minister (ODPM), How to choose choice, Londres, ODPM, 2002.

${ }^{16} \mathrm{BCC}$ Housing department, BME communities' access to outer city housing, Birmingham, BCC, 1998.
} 
intégrant fortement la discrimination et sa dimension institutionnelle. La Commission départementale d'accès à la citoyenneté, Codac, a également échoué à promouvoir une représentation locale partagée du problème des discriminations dans l'accès au logement. La construction dominante du problème, en phase avec le cadrage national universalisant, est celle de «l'exclusion sociale». Aussi les quelques dispositifs déployés au plan local, tels que le Plan départemental pour le logement des personnes défavorisées (loi Besson) ou l'Accord collectif départemental (qui fixe, pour chaque organisme HLM, un objectif annuel quantifié d'accueil de ménages en difficulté) visent-ils les «personnes en difficulté » ou « défavorisées », définies par leur situation économique et sociale, sans spécifier les minorités ethniques. L'absence de tout moyen statistique d'objectivation des inégalités ethniques entretient le déni des discriminations. Le paradoxe fondamental est que les discours des acteurs que nous avons interviewés au sein des organismes HLM abondent de références à l'appartenance ethnique des candidats ou locataires. Comment expliquer ce paradoxe ? Peut-on déceler des mécanismes de racisme institutionnel dans le logement social à Marseille ? Dans quelle mesure ces mécanismes sont-ils proches de ceux identifiés à Birmingham ?

\section{La vigueur paradoxale du racisme institutionnel}

Une première similitude avec le cas de Birmingham est le conflit d'objectifs qui tiraille les organismes HLM, amenés à jongler entre leur mission sociale d'accueil des plus démunis et leurs contraintes de gestion (limiter la vacance et les coût de gestion des ensembles). Le poids de ces contraintes est tel que les pratiques d'attribution sont orientées par un objectif de réduction du risque, qu'il soit financier, politique ou sociologique ${ }^{18}$. Mais dans le cas français, ces contradictions entre objectifs officiels et officieux sont renforcées par la contradiction entre les deux principaux objectifs officiels eux-mêmes, à savoir le droit au logement et la mixité sociale. On touche là une différence essentielle avec le cas britannique: en France, la concentration ethnico-résidentielle (la «ghettoïsation ») est perçue comme un problème, une menace à l'intégrité de la République et à l'unité de son territoire, et les acteurs des attributions se voient reconnaître une mission d'ingénierie sociale et, implicitement, ethnique. Ces injonctions «en creux» soulèvent une contradiction fondamentale dans le contexte d'une politique nationale qui refuse toute reconnaissance explicite d'une dimension ethnique des problèmes d'accès au logement. En outre, droit au logement et mixité sociale ne sont pas aisément conciliables : si le principe de droit au logement suppose d'accueillir les personnes les plus en difficulté, la structure du stock de logements sociaux est telle que les logements les plus abordables sont concentrés dans certains quartiers. Comment dès lors accueillir les plus démunis sans les concentrer dans certains espaces urbains? Dans leurs politiques et pratiques

${ }^{17}$ C. Bourgeois, L'Attribution des logements sociaux. Politique publique et jeu des acteurs locaux, Paris, L'Harmattan, 1996. 
d'attribution, les acteurs HLM gèrent les contradictions et le flou des objectifs nationaux d'action publique tout en intégrant leurs propres préoccupations de gestion. Les acteurs font ainsi constamment référence à la «gestion fine», au cas par cas, des attributions, ce degré de finesse étant apprécié à l'aune de deux conditions. Faire une attribution «fine», c'est d'abord savoir faire la différence entre un «bon» et un «mauvais» candidat; c'est ensuite savoir mettre le «bon» candidat au «bon endroit». Ces deux impératifs génèrent une retraduction, dans l'univers de sens des acteurs, des catégories officielles de l'action publique, le droit au logement et la mixité sociale.

C'est là qu'interviennent les catégorisations et stéréotypes ethniques. Ces stéréotypes englobent des préjugés relatifs à trois principaux éléments : la qualité de bon payeur, mais encore plus la capacité à habiter un logement et davantage encore la capacité à s'intégrer dans son environnement. Est ici construite comme particulièrement problématique la figure du «Comorien », particulièrement saillante dans le discours des acteurs marseillais. La construction stéréotypique du groupe «Comoriens » procède toujours du même point de départ: le présupposé d'une structure familiale ingérable. Cette structure familiale est renvoyée à la culture comorienne, qui «n'est pas toujours évidente ». Cette différence culturelle est en outre essentialisée, renvoyée à un déterminisme naturel, biologique : «c'est quasiment dans leurs gènes ». Le constat - socialement construit - de la différence culturelle débouche sur celui de l'inadaptation des Comoriens au logement. Toute famille comorienne est considérée comme déterminée à reproduire les difficultés soulevées par des familles comoriennes précédemment logées dans le parc. Toute possibilité de différences individuelles au sein du groupe est abolie : l'individu n'est que l'expression du groupe, de ses traits culturels essentialisés. La possibilité ou la nécessité de prévoir un accompagnement social dans le logement pour les primo-arrivants n'est ainsi pas évoquée. La seule solution considérée par les acteurs consiste à refuser les familles comoriennes, ou au moins à éviter leur cohabitation avec d'autres communautés. A l'inverse, les Arméniens sont définis par un ensemble de traits culturels positifs : une structure familiale stable ; un désir d'intégration sociale, notamment par le travail ; le respect des engagements, en particulier pour le paiement des loyers. Leur supposée capacité d'intégration leur fournit une image de «bons locataires ». Mais de façon générale, le locataire «normal», désirable, appartient de toute évidence à la majorité ethnique : il est présent, dans le discours des acteurs, sous les expressions «Français de souche », "parfaits Français", ou encore "salarié lambda, très européanisé ». Derrière ces stéréotypes, négatifs ou positifs, apparaît une considération centrale, celle de l'écart à la norme culturelle $^{19}$. La construction sociale de la «distance culturelle» conduit les acteurs à comparer

\footnotetext{
${ }^{18}$ P. Simon, «Le logement social en France et la gestion des 'populations à risques' », $H \& M, \mathrm{n}^{\circ} 1246$, novembre-décembre, 2003, pp. 76-91.

${ }^{19}$ Dans le néoracisme ou racisme culturel, la thématique de la différence culturelle, présumée essentielle, a remplacé celle de la lutte entre les races. Cf. P.-A. Taguieff, La Force du préjugé. Essai sur le racisme et ses doubles, Paris, La Découverte, 1988 ; M. Barker, The New Racism, Londres, Junction Books, 1981.
} 
l'adaptabilité supposée des différents groupes ethnicisés et de là à favoriser plus ou moins leur accès au logement.

C'est ensuite au nom de ces barrières culturelles que sont légitimées différentes pratiques liées à la gestion des «équilibres ». D'une part, des limites sont fixées à l'accueil de certains groupes ethniques sur certains ensembles ou cages d'escalier, au nom du respect d'un supposé «seuil de tolérance $»^{20}$. D'autre part, est organisée la concentration de certains groupes ethniques sur certains territoires, afin d'éviter de disséminer des populations labellisées « à risques » dans l'ensemble du parc et d'éviter la co-présence des cultures présumées inconciliables, au prix d'une remise en cause radicale du bien-fondé d'un principe de mixité sociale. Ces deux orientations (seuil de tolérance et concentration ethnico-spatiale) ne sont contradictoires qu'en apparence, car elles ne s'appliquent pas aux mêmes territoires : c'est pour préserver l'attractivité des ensembles et quartiers les plus attractifs qu'on y fixe des seuils de tolérance et qu'on concentre des groupes ethniques minoritaires dans les cités les plus dégradées, qu'on laisse «pourrir », pour reprendre le terme du jargon HLM.

Une double conclusion peut être formulée. La comparaison entre Birmingham hier et aujourd'hui, tout d'abord, conduit à pointer les limites des politiques anti-discrimination développées depuis les années 1980. Les politiques antiracistes ont laissé intact le cœur des politiques d'attributions, notamment le conflit entre objectifs sociaux et manageriaux. En solidifiant l'idée d'une spécificité des problèmes des minorités ethniques, elles ont même contribué à essentialiser la «différence culturelle». Pourtant, on ne peut se limiter à ces constats négatifs. Ces politiques ont produit des transformations substantielles des représentations et des pratiques. Elles ont contribué à la reconnaissance générale de l'existence du racisme institutionnel, même si celui-ci demeure euphémisé, dissimulé derrière les invocations des «besoins spécifiques » d'une part et du racisme individuel d'autre part. Elles ont aussi très certainement un rôle dans l'effacement des stéréotypes ethniques les plus négatifs. Si les discours de acteurs révèlent une certaine essentialisation de la différence culturelle, ils véhiculent très peu de stéréotypes explicites sur l'inadaptation des immigrés ou le choc des cultures. Aujourd'hui, à la différence des résultats de Henderson et Karn, les acteurs ne font pas constamment référence au mérite et à la respectabilité des ménages, et ils apprécient encore moins cette respectabilité à l'aune de l'appartenance ethnique. Reste à savoir si l'effacement de ces considérations du discours des acteurs révèle leur effacement effectif des pratiques ou plutôt un seul effet de censure.

\footnotetext{
${ }^{20}$ Sur cette notion et ses présupposés, voir V. De Rudder, «'Seuil de tolérance' et cohabitation pluriethnique », in P.-A. Taguieff, dir., Face au racisme, Paris, La Découverte, 1991.
} 
La comparaison entre Marseille et Birmingham fait en revanche ressortir la force et l'épaisseur des stéréotypes ethniques mobilisés par les acteurs marseillais, ce qui peut sembler paradoxal au pays de l'intégration républicaine. L'expérience marseillaise tient dans ce paradoxe d'un déni généralisé des discriminations alors même que les représentations des acteurs des attributions sont très fortement ethnicisées. Si le racisme institutionnel repose ici sur des mécanismes en partie similaires à ceux observés à Birmingham (forte discrétion des bailleurs sociaux dans la définition de leurs stratégies, contradictions entre objectifs sociaux et de gestion) il se fonde aussi sur des mécanismes originaux, à commencer par la contradiction bien française entre les principes de droit au logement et de mixité sociale. En l'absence de politique de lutte contre les discriminations, ces contradictions nourrissent des stéréotypes abondants sur les groupes «d'origine immigrée » et les désavantagent dans le processus d'attribution. Au final, cette traversée du miroir montre l'emprise des idéologies nationales sur les mises en formes des problèmes et les politiques formulées, mais aussi la forte présence d'un racisme institutionnel des deux côtés de la Manche. 